\title{
Microscale Fluid Mechanics of Making Thin Specimens for Cryo-EM
}

\author{
Robert M. Glaeser ${ }^{1 *}$, Maxim Armstrong ${ }^{2}$, Bong-Gyoon Han $^{1}$ and Daniel A. Fletcher ${ }^{2}$ \\ 1. MBIB Division, Lawrence Berkeley National Lab, Berkeley, CA, USA. \\ 2. Bioengineering Dept., Univ. of California Berkeley, Berkeley, CA, USA. \\ * Corresponding author: rmglaeser@1bl.gov
}

The standard method of making thin specimens for single-particle electron cryo-microscopy (cryo-EM) involves blotting excess sample by pressing filter paper against one or both sides of the specimen grid (EM grid), and then rapidly freezing (vitrifying) the resulting thin film of water that remains on the grid [1]. A shortcoming of this method is that the thickness of the remaining sample is much too variable from one grid to the next, and even over the area of a single grid. A not atypical example of the ice-thickness variation is seen in the TEM "grid atlas" shown in Figure 1, which was recorded with Leginon [2]. As a result of such variability, one might have to prepare and screen a number of grids before one is found for which the ice thickness is suitable, over an acceptable fraction of the area, for high-resolution data collection. Here we seek to improve our understanding of how thin films of aqueous sample are produced by blotting with the commonly used Vitrobot instrument. The expectation is that a better understanding will point to ways that can improve the standard method, or that it may even inform the development of alternative approaches.

Although blotting has been the established method of preparing cryo-EM grids for over 30 years, there still is little theoretical understanding or direct observation of what occurs during this process. Here we use a combination of:

(1) cryo-SEM images of grids prepared previously for single-particle cryo-TEM, which reveal that areas of thick ice consist mainly of localized puddles on the front of the grid, while menisci that are seen, not uncommonly, near the grid bars exist on the back, wetting the 90-degree corner formed by grid bars and the overlying carbon film;

(2) confocal light microscopy of a model of the early stage of blotting, which reveals that the welllubricated gap between filter paper and grid is much thicker than the final, vitrified sample; and

(3) high-speed Reflection Interference Contrast Microscopy (RICM) of a model of later stages of the thinning process, which captures the rapid intrusion of fingers of air from the perimeter, into the gap, explaining how excess sample is removed.

Figure $2 \mathrm{~A}$ shows a schematic diagram of how the RICM images were produced, and Figures $2 \mathrm{~B}$ and $2 \mathrm{C}$ are examples of images recorded at different times after blotting began.

These experimental observations are interpreted in accord with the formalism of microscale fluid mechanics. From this perspective, both fluid flow and fluid retention are governed by the boundary conditions that exist in a given situation. The variable outcome between one instance of blotting and the next, and between one area of a grid and the next, are thus attributed to the highly variable boundary conditions imposed when, in each instance, irregular patterns of contact are made between fibers of the filter paper and the EM grid. One path forward, to achieve more uniform and more reproducible results, might thus be to impose more consistent boundary conditions, such as removing solution symmetrically from the perimeter but not over the face of the grid, similar to what has been done in experiments that characterize the disjoining pressure exerted between interfaces of thin liquid films [3, 4]. 


\section{References:}

[1] MJ Dobro et al., in "Methods in Enzymology: Cryo-Em, Part a", GJ Jensen (Ed.) p 63.

[2] C Suloway et al., Journal of Structural Biology 151 (2005), p. 41.

[3] KJ Mysels, The Journal of Physical Chemistry 68 (1964), p. 344.

[4] This work was supported in part by NIH grant GM051487. We especially thank John Turner for help when using the cryo-SEM instrument at NCEM within LBNL.
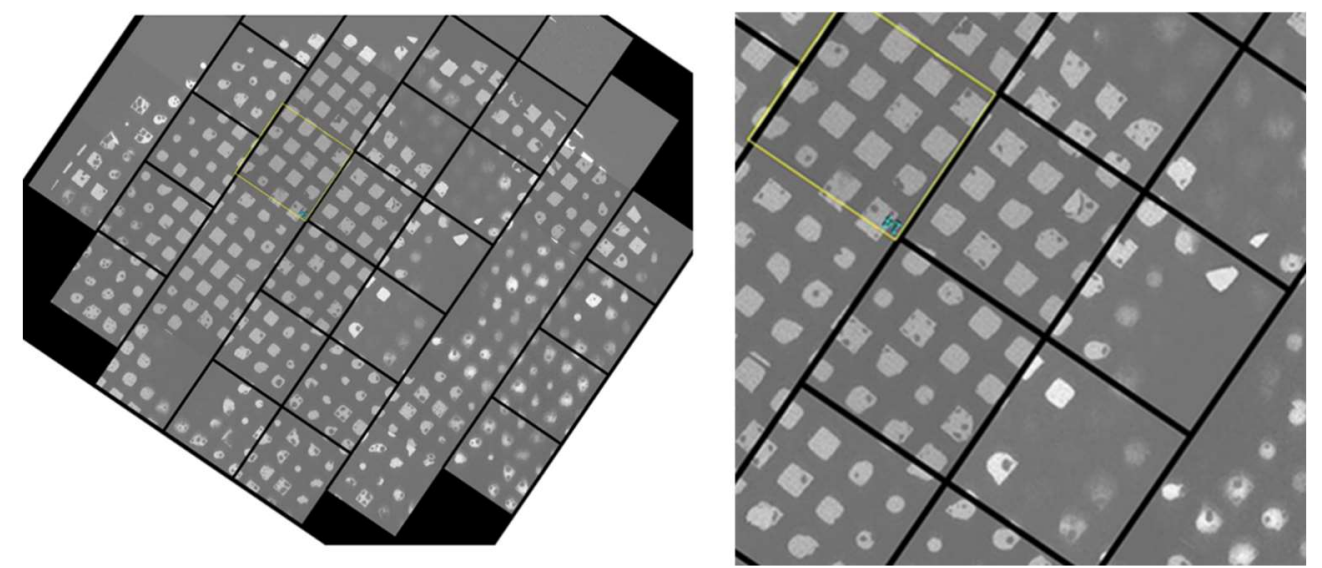

Figure 1.

representative example of a so-called grid atlas showing variations in electron transparency of the specimen due to variations in the vitrified-ice thickness.
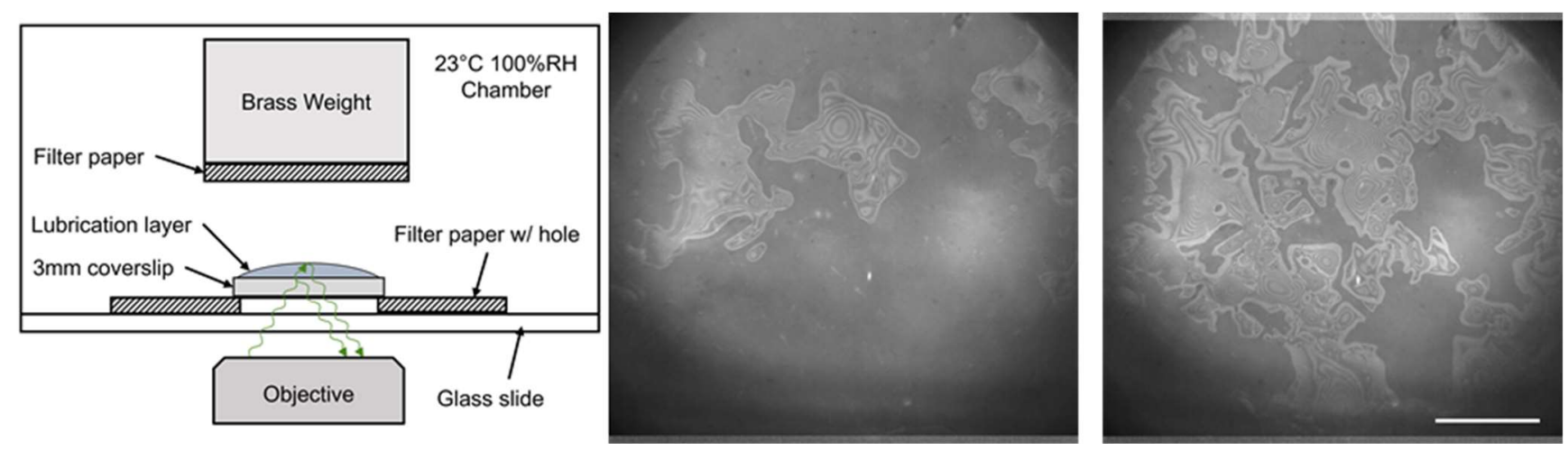

Figure 2. Schematic diagram (left) of the equipment used to obtain high-speed RICM images during blotting of a $3 \mathrm{~mm}$ diameter glass cover slip with a force similar to that in a Vitrobot, and two frames of a movie showing the intrusion of fingers of air from the perimeter of the cover slip. The middle panel shows partial removal of water after $700 \mathrm{~ms}$ and the right-hand panel shows the nearly final state achieved after $1200 \mathrm{~ms}$. The scale bar in the right-hand panel corresponds to $500 \mu \mathrm{m}$. 